\title{
Tratamiento ambulatorio de la diverticulitis aguda no complicada
}

\author{
Ana Sánchez Gollarte, ${ }^{1}$ Ricardo Alvarado Hurtado, ${ }^{1}$ Cristina Vera Mansilla, ${ }^{1}$ Fernando Mendoza \\ Moreno, ${ }^{1}$ Manuel Díez Alonso, ${ }^{1}$ Francisca García-Moreno Nisa ${ }^{1,2}$ \\ ${ }^{1}$ Servicio de Cirugía General y del Aparato Digestivo, Hospital Universitario Príncipe de Asturias, Alcalá de Henares. \\ ${ }^{2}$ GRUPO GITBIT-UAH CIBER-BBN, departamento de Cirugía y Especialidades Médicas y Sociales de la Universidad de Alcalá. \\ Madrid, España.
}

Acta Gastroenterol Latinoam 2020;50(4):439-444

Recibido: 02/06/2020 / Aceptado: 09/11/2020 / Publicado online: 14/12/2020

\section{Resumen}

Introducción. La diverticulitis aguda es una complicación importante de la enfermedad diverticular. La antibioterapia se ha considerado clásicamente el pilar del tratamiento de estos pacientes. El ánimo de este estudio es presentar los resultados del protocolo del tratamiento ambulatorio de la diverticulitis aguda leve en nuestro centro, valorar sus resultados y conocer si es necesario modificar dicho protocolo en base a la evidencia en el momento actual. Material y métodos. Análisis retrospectivo de los pacientes incluidos en el protocolo de tratamiento ambulatorio de la diverticulitis aguda no complicada en nuestro centro en el periodo comprendido entre el 1 de enero y el 31 de diciembre de 2018. Se
Correspondencia: Francisca García-Moreno Nisa GRUPO GITBIT-UAH CIBER-BBN, departamento de Cirugia y Especialidades Médicas y Sociales de la Universidad de Alcalá, Campus Universitario 28805. Alcalá de Henares, Madrid, España Tel.: +34666326521

Correo electrónico: francisca.garciam@uah.es analizó la presencia de complicaciones en el mismo episodio, la necesidad de ingreso y la tasa de recidiva hasta diciembre de 2019. Finalmente se estudiaron los informes de las colonoscopias realizadas tras la resolución del proceso y la necesidad o no de tratamiento quirúrgico. Resultados. Un total de 64 pacientes fueron incluidos en el protocolo de ambulatorización representando el $68 \%$ del total de las diverticulitis tratadas. Durante el seguimiento, 6 enfermos requirieron ingreso $(9,3 \%)$ en los días posteriores. Del total de pacientes estudiados, 5 (7,8\%) presentaron recidiva a lo largo de los años 2018-2019: sólo una de ellas fue una recidiva grave y precisó tratamiento quirúrgico. Los datos de nuestro estudio indican que el tratamiento ambulatorio de la diverticulitis aguda leve en nuestro medio es seguro con el protocolo actual, ya que el $90,7 \%$ fue tratado con éxito.

Palabras claves. Enfermedad diverticular, antibióticos, diverticulitis aguda no complicada.

\section{Outpatient Treatment of Uncomplicated Acute Diverticulitis}

\section{Summary}

Introduction. Acute diverticulitis is a major complication of diverticular disease. Antibiotic therapy has classically been considered the mainstay of treatment for these patients. The aim of this study is to present the results of the outpatient treatment protocol for mild acute diverticulitis in our cen- 
tre, assess its results and understand if it is needed to modify said protocol based on the current evidence. Material and methods. Retrospective analysis of the patients included in the outpatient treatment protocol for uncomplicated acute diverticulitis in our centre in the period between the 1st of January and the 31 of December of 2018. The presence of complications in the same episode, the need for admission and recurrence rate until December 2019. Finally, the reports of the colonoscopies performed after the resolution of the process and the need or not for surgical treatment were studied. Results. A total of 64 patients were included in the outpatient protocol, representing $68 \%$ of the total of diverticulitis treated. During follow-up 6 patients required admission (9.3\%) in the following days. Of the total number of patients studied, 5 (7.8\%) presented recurrence throughout the years 2018-2019, only one of them was a severe recurrence and required surgical treatment. The data from our study indicate that the outpatient treatment of mild acute diverticulitis in our setting is safe with the current protocol, since $90.7 \%$ were treated successfully.

Key words. Diverticular disease, antibiotics, acute uncomplicated diverticulitis.

\section{Abreviaturas}

EDC: Enfermedad diverticular de colon.

TAC: Tomografia axial computarizada.

PCR: Proteina C Reactiva.

\section{Introducción}

La enfermedad diverticular del colon (EDC) ocurre fundamentalmente en los países desarrollados y su incidencia está aumentando ${ }^{1}$ en los países asiáticos y del este debido a los cambios en la dieta. En estas áreas geográficas, progresivamente, se han sustituido en la dieta los alimentos ricos en fibra por los alimentos procesados. Este dato epidemiológico apoya la hipótesis de que la etiología es multifactorial y, que además de existir una predisposición genética, los factores ambientales y dietéticos representan las principales causas para su desarrollo. La dieta pobre en fibra, a favor del consumo de harinas refinadas y azúcares, es un aspecto esencial. La ingesta de fibra aumenta el bolo fecal, disminuye la presión intracólica y acelera el tránsito intestinal. ${ }^{2}$ En general, la EDC afecta por igual a ambos sexos con una edad media de presentación entorno a los 60 años. Se clasifica en tres entidades clínicas diferentes: enfermedad asintomática, enfermedad sintomática no complicada y enfermedad complicada. ${ }^{3}$
La mayoría de los pacientes con diverticulosis no presenta síntomas y el diagnóstico suele ser un hallazgo incidental. Aproximadamente el 10\% de ellos puede referir síntomas derivados del trastorno funcional motor existente en el colon. La clínica es inespecífica y caracterizada por dolor abdominal cólico, moderada distensión abdominal, anorexia, flatulencia y alteración del ritmo deposicional. El dolor suele localizarse en la fosa iliaca izquierda, es de intensidad variable y se presenta como una crisis que dura de uno a varios días, coincidiendo (habitualmente) con un periodo de estreñimiento. Se incrementa después de las comidas y se alivia tras la defecación o la expulsión de gases. ${ }^{4}$

La diverticulitis es una complicación importante de la enfermedad diverticular. Aunque la mayoría de los episodios es no complicada (70\%) y responde bien al tratamiento médico conservador, pueden desarrollarse complicaciones como absceso pericólico, absceso pélvico o perforación intestinal con peritonitis purulenta o incluso fecal., 6

Los pacientes con cuadros leves y estadio Hinchey I se pueden tratar de forma ambulatoria con una dieta líquida y antibioterapia oral de 7 a 10 días: con una tasa de éxito en torno al 97\%, manifiestan mejoría a las 72 horas del inicio del tratamiento, según la guía de la World Gastroenterology Organization.

La antibioterapia se ha considerado clásicamente el pilar del tratamiento de estos pacientes, aunque se especula que la inflamación crónica se debe a una alteración en la microflora normal del colon y que esta es distinta en los pacientes con baja ingesta de fibra. Por este motivo, se ha considerado que la utilización de los fármacos probióticos en el tratamiento médico de los pacientes con diverticulosis sintomática es de utilidad. ${ }^{8}$ Por otra parte, en el momento actual, se cuestiona que la antibioterapia sea útil en los pacientes con diverticulitis leve, ya que se ha sugerido que se trate más bien de un tipo de enfermedad inflamatoria intestinal no debida a una microperforación. ${ }^{8}$ Una revisión reciente encontró que sólo el $9 \%$ de los gastroenterólogos y el 10\% de los cirujanos consideran necesaria la administración de antibioterapia oral en los casos leves de diverticulitis aguda. ${ }^{?}$

El ánimo de este estudio es presentar los resultados del protocolo del tratamiento ambulatorio de la diverticulitis aguda leve en nuestro centro, comparándolos con la evidencia existente y conocer si es necesario modificarlo.

\section{Material y métodos}

Análisis retrospectivo de los pacientes incluidos en el protocolo del tratamiento ambulatorio de la diverticulitis aguda no complicada en nuestro centro en el periodo 
comprendido entre el 1 de enero y el 31 de diciembre de 2018. Se analizó la presencia de complicaciones en el mismo episodio, la necesidad de ingreso y la tasa de recidiva hasta diciembre de 2019. Se incluyeron datos demográficos referentes a la edad, el sexo y los antecedentes personales de los pacientes. Como datos referentes al proceso inflamatorio se tuvieron en cuenta los valores analíticos de leucocitos y PCR, los hallazgos de la TAC y la necesidad o no del tratamiento antibiótico intravenoso. Finalmente, se estudiaron los informes de las colonoscopias realizadas tras la resolución del proceso y la necesidad o no de un tratamiento quirúrgico.

Ante la sospecha de una diverticulitis aguda (dolor en la fosa iliaca izquierda con signos locales de irritación peritoneal, asociado a malestar general y rectorragia o estreñimiento), en el servicio de urgencias se le realiza a cada paciente una tomografía axial computarizada (TAC) abdominal con contraste oral e intravenoso y analítica urgente incluyendo bioquímica, hemograma, estudio de coagulación PCR y hemocultivos en caso de fiebre alta (temperatura axilar superior a 38,5 ${ }^{\circ} \mathrm{C}$ ). De acuerdo con la guía clínica de cirugía colorrectal de la Asociación Española de Cirujanos, consideramos diverticulitis aguda no complicada a aquella que no asocie sepsis generalizada, absceso, fistula, oclusión intestinal o una perforación libre. Se utilizó la clasificación de Ambrosetti/ Doringer-Neff ${ }^{10}$ para valorar la gravedad y los criterios del American College of Chest Physicians y de la Society of Critical Care Medicine ${ }^{11}$ para la presencia de sepsis. Se consideraron criterios de inclusión y exclusión para el tratamiento ambulatorio de la diverticulitis aguda los reflejados en la tabla 1 .

El paciente que cumplía los criterios de inclusión del protocolo recibía indicaciones para el tratamiento dietético y antibiótico domiciliario. Se garantizó una correcta administración del fármaco vía oral durante el tiempo previsto dado que debían presentar una correcta tolerancia oral y un entorno familiar favorable para ser incluidos en este protocolo de tratamiento. Los pacientes recibieron 1 dosis cada 8 horas de amoxicilina ácido clavulánico (875/125 mg) durante 7 a 10 días. Los pacientes alérgicos a penicilinas recibieron ciprofloxacino $500 \mathrm{mg}$ cada 12 horas y metronidazol $500 \mathrm{mg}$ cada 8 horas durante 7 a 10 días. Las recomendaciones dietéticas se incluyen en la tabla 2. Se consideró un fracaso del tratamiento ambulatorio la necesidad de ingreso hospitalario para la administración intravenosa del antibiótico o analgesia, así como la necesidad de cirugía urgente.

Para el seguimiento, se facilitó a los pacientes una hoja informativa que indicaba claramente la naturaleza de su proceso, así como las recomendaciones del tratamiento, la dieta, el seguimiento y los signos de alarma ante los que debían consultar al servicio de urgencias. Los pacientes debían tomarse la temperatura a la mañana y a la tarde. Si tenían una temperatura mayor o igual a $38{ }^{\circ} \mathrm{C}$ debían acudir al servicio de urgencias, así mismo si el dolor abdominal aumentaba o no cedía con un gramo de paracetamol oral, se sentían mal (presentaban afectación del

Tabla 1. Criterios de inclusión y exclusión de pacientes en el protocolo de tratamiento ambulatorio de la diverticulitis aguda leve

\begin{tabular}{|c|c|}
\hline Criterios de inclusión & Criterios de exclusión \\
\hline Aceptación del paciente. & No aceptación del paciente. \\
\hline Edad $>18$ y $<80$ años. & $\begin{array}{l}\text { Paciente inmunodeprimido } \\
\text { o diabético. }\end{array}$ \\
\hline $\begin{array}{l}\text { Hallazgos en la TAC: } \\
\text { - Estadio la e lb. } \\
\text { - Diverticulitis leve. } \\
\text { - No perforación ni absceso. }\end{array}$ & $\begin{array}{l}\text { Hallazgos en la TAC: } \\
\text { - Diverticulitis complicada o grave. } \\
\text { - Estadios Ic a IV. }\end{array}$ \\
\hline No toma corticoides & Toma de corticoides \\
\hline 0 inmunosupresores. & 0 inmunosupresores. \\
\hline $\begin{array}{l}\text { Tolerancia oral } \\
\text { (no vómitos). }\end{array}$ & $\begin{array}{l}\text { Intolerancia oral } \\
\text { (vómitos). }\end{array}$ \\
\hline $\begin{array}{l}\text { Sin signos de sepsis grave: } \\
\text { - Hipotensión (TAS < } 90 \text { mmHg). } \\
\text { - Hipoperfusión: acidosis láctica, } \\
\text { oliguria, alteración aguda del nivel } \\
\text { de conciencia. }\end{array}$ & Con signos de sepsis grave. \\
\hline $\begin{array}{l}\text { Residencia en el área de atención } \\
\text { domiciliaria. }\end{array}$ & $\begin{array}{l}\text { Lugar de residencia fuera del área } \\
\text { de atención domiciliaria. }\end{array}$ \\
\hline Cobertura sociofamiliar adecuada. & Comorbilidad asociada importante. \\
\hline
\end{tabular}

Tabla 2. Recomendaciones dietéticas para el tratamiento ambulatorio de las diverticulitis agudas leves recogidas en el protocolo de tratamiento

Del primer al tercer día:

- Beber al menos un litro y medio de bebida isotónica al día.

- Beber agua e infusiones libremente.

- No tomar alimentos sólidos hasta la siguiente revisión.

Del cuarto al séptimo día:

- Dieta blanda (queso fresco, yogurt, leche desnatada, arroz blanco, natillas, arroz con leche).

\section{A partir del séptimo día:}

- Si se encuentra claramente mejor, dieta mediterránea rica en frutas, verduras y legumbres. 
estado general o decaimiento), o presentaban escalofríos. A los 5 y 10 días del diagnóstico, se evaluó a cada paciente en consultas externas del Servicio de Cirugía General con evaluación clínica y analítica. Si durante el seguimiento ambulatorio el paciente empeoró o no había una mejoría clara en su estado, se indicó el ingreso hospitalario para comenzar el tratamiento antibiótico intravenoso. Tras la resolución del proceso agudo, el paciente fue remitido a la consulta externa en un plazo de un mes para realizar un estudio con colonoscopia y la TAC.

\section{Resultados}

Un total de 64 pacientes fue incluido en el protocolo de ambulatorización en el periodo de tiempo estudiado (36/28 varón/mujer, edad media de 56 años), representando el $68 \%$ del total de diverticulitis tratadas. Solo 5 pacientes tenían historia previa de diverticulitis aguda. El tiempo medio de desarrollo de los síntomas hasta consultar al servicio de urgencias fue de 2,3 días (Rango: 1-5). La adherencia al protocolo fue alta: todos los pacientes siguieron el protocolo de tratamiento. El valor medio de la PCR al diagnóstico fue de 79,2 (Rango: 5-223) y recuento leucocitario medio de 9800 cels $/ \mathrm{mm}^{3}$ (Rango: 4556-21300). Respecto a los hallazgos de la TAC, de los 64 pacientes, 38 se encontraban en estadio Ia y 26 estadio Ib de la escala de Ambrosetti.

Durante el seguimiento 6 (5/1 varón/mujer) enfermos requirieron ingreso (6/64) en los días posteriores. Entre estos, en 5 (5/64) se debió a persistencia de la clínica tras concluir el tratamiento, aunque sin desarrollar complicaciones graves. En el otro caso (1/64), en la revisión al $5^{\circ}$ día del inicio del tratamiento ambulante, el paciente refería dolor abdominal y febrícula, por lo que se remitió al servicio de urgencias para su estudio. En la TAC se objetivó la presencia de múltiples colecciones intraabdominales que precisaron drenaje y lavado por abordaje laparoscópico. La evolución postoperatoria fue satisfactoria. Todos los pacientes que precisaron ingreso o cirugía presentaron un tiempo de evolución de los síntomas antes de consultar a urgencias (y de iniciado el tratamiento) superior a 3 días.

Los 58 pacientes que no precisaron ingreso hospitalario durante el seguimiento ambulatorio fueron evaluados en una consulta al mes del inicio del cuadro, realizándose una exploración clínica, analítica, colonoscopia y la TAC. En 5 (5/58) de los pacientes no se realizó la colonoscopia al mes por mala preparación (puntuación en escala de Boston inferior a 6), realizándose en las 4 semanas siguientes. En el estudio realizado tras la resolución del cuadro agudo, en dos pacientes (2/58) se detectó un adenocarcinoma de colon, por lo que precisaron una sigmoi- dectomía programada (estadios tumorales T3N0 y T4N1 en el estudio final de anatomía patológica).

Del total de pacientes estudiados, 5 (5/64) presentaron recidiva a lo largo de los años 2018-2019. Solo una de ellas fue una recidiva grave y precisó tratamiento quirúrgico. Los pacientes que presentaron recidiva tenían un Ambrosetti Ib (Tabla 3).

Tabla 3. Resultados

Varón/mujer: 36/28

PCR: 79,2 (Rango 5-223).

Recuento leucocitario: 9800 cels/mm³ (Rango: 4556-21300).

TAC: Ambrosetti la (38), lb (26).

Ingreso hospitalario: 6/64.

Intervención quirúrgica:1/64.

Colonoscopia ambulante al mes del cuadro: 53/58.

Presencia de un tumor en la colonoscopia de seguimiento: 2/58.

Recidiva a los 2 años: 5/64.

\section{Discusión}

La diverticulitis aguda debe ser tratada inicialmente con reposo digestivo, tratamiento antibiótico y analgesia. Los pacientes que no presentan indicación de cirugía urgente pueden ser tratados mediante ingreso hospitalario o en régimen ambulatorio. El $80 \%$ de los episodios de diverticulitis aguda se asocia a la existencia de un flemón con reacción mesentérica local. La posibilidad de realizar un diagnóstico correcto por la TAC, descartando otras patologías y estableciendo el grado de complicación de la enfermedad, permite emplear el tratamiento ambulatorio en la mayor parte de las diverticulitis leves con un elevado margen de seguridad. ${ }^{12}$ El tratamiento domiciliario, mediante la ingesta de líquidos, antibióticos y analgesia oral, está condicionado por el estado general del paciente, su edad y la patología médica asociada. Es fundamental que haya buena colaboración por parte del mismo, un entorno familiar adecuado y la posibilidad de realizar un control ambulatorio estricto. ${ }^{13}$

En esta línea, Cirocchi et al. ${ }^{14}$ concluyen tras una revisión sistemática y metaanálisis que el tratamiento ambulatorio es seguro cuando se selecciona correctamente el grupo al que va dirigido, no siendo la localización de la diverticulitis un criterio de selección para el tratamiento ambulatorio. Los parámetros que no demostraron influir en la tasa de fracaso del tratamiento ambulante fueron: la 
presencia de episodios previos de diverticulitis, comorbilidades, presencia de aire pericólico, absceso intraabdominal y tipo de antibioterapia o diabetes.

Solo se ha determinado que el sexo femenino y la presencia de líquido libre en la TAC son las variables predictoras del fallo en la ambulatorización de este proceso. ${ }^{12}$

Desde la última década, ya aparecen publicaciones indicando que es correcto restringir el uso de la antibioterapia en los pacientes seleccionados con diverticulitis aguda no complicada. Así se posiciona la Guía de la Asociación Americana de Gastroenterólogos que recomienda que los antibióticos no sean empleados de forma rutinaria en esta patología. 15,16

En 2017, Daniels et al. ${ }^{17}$ publicaron un ensayo clínico randomizado realizado en Holanda en el que se incluyeron 528 pacientes bajo tratamiento antibiótico intravenoso $\mathrm{u}$ oral según la tolerancia del paciente o solo observación. Solo se incluyeron, como en nuestro protocolo, pacientes con estadio Ia-Ib de la escala de Ambrosetti y sin sepsis en el primer episodio de diverticulitis aguda. Estudiaron, como objetivo primario, el tiempo de recuperación en los primeros seis meses tras el diagnóstico y, como secundarios, la tasa de reingresos hospitalarios, las complicaciones, la recurrencia y mortalidad, sin encontrar que la observación domiciliaria frente al tratamiento antibiótico prolongue el tiempo de recuperación y considerando que es una opción correcta en los pacientes seleccionados. Stollman et al. ${ }^{16}$ recomiendan el uso de antibioterapia en los pacientes inmunocomprometidos (embarazadas, pacientes con enfermedades renales crónicas, en tratamiento con corticoides o con enfermedades del colágeno) y pacientes sépticos debido a una evolución más grave por su alteración en la respuesta inmune. En este sentido, los resultados del ensayo clínico randomizado multicéntrico, que está desarrollando en España The Diverticulitis Study Group, ayudarán a aclarar que pacientes en nuestro medio se beneficiarían de recibir antibioterapia. ${ }^{18}$

En el año 2018, Emile et al. ${ }^{19}$ publicaron una revisión sistemática de la evidencia existente hasta ese entonces sobre la eficacia del tratamiento sin antibióticos de la diverticulitis aguda y su tasa de complicaciones y recurrencias frente al tratamiento médico habitual. En su análisis se incluyeron 2565 pacientes, de los cuales el 65,1\% fueron tratados sin antibioterapia. En el metaanálisis no encontraron diferencias estadísticamente significativas entre los grupos con y sin tratamiento antibiótico en lo que se refiere a la tasa de fallo del tratamiento $(O d d$ s ratio $=1,5$, $p=0,06)$, la recurrencia del cuadro (Odds ratio $=0,81$, $p=0,2)$, las complicaciones (Odds ratio $=0,56, p=0,25$ ), los ingresos (Odds ratio $=0,97, p=0,91)$, la necesidad de intervención quirúrgica (Odds ratio $=0,59, p=0,28) \mathrm{y}$ la mortalidad (Odds ratio $=0,64, p=0,47)$. Finalmente, concluyeron que el tratamiento ambulatorio de la diverticulitis no complicada es posible, seguro y efectivo. Añadir un tratamiento antibiótico oral de amplio espectro no sirve para disminuir la tasa de fallo del tratamiento, las recurrencias, las complicaciones, los ingresos hospitalarios y la necesidad de cirugía.

También existen experiencias negativas respecto al manejo sin antibioterapia no referidas a la eficacia, ni eficiencia del mismo, sino a la adherencia de los pacientes. Azhar et al. ${ }^{20}$ han publicado recientemente sus dificultades para introducir un nuevo protocolo de tratamiento en sus prácticas clínicas, con una aceptación por parte de los pacientes más baja de la esperada. Insisten en que es preciso una buena selección de los pacientes de cara a instaurar estos protocolos de tratamiento, términos en los que estamos de acuerdo.

En cualquier caso, las ventajas son de sobra conocidas. En el año 2013, Lorente et al..$^{21}$ ya pusieron de manifiesto que el tratamiento ambulatorio de la diverticulitis aguda no solo es seguro, sino que también reduce más de un $60 \%$ los costes sanitarios. En su estudio, no hubo diferencias en el porcentaje de fracaso del tratamiento entre los grupos de tratamiento ambulatorio y de ingreso hospitalario. Van Dijk et al. ${ }^{22}$ en una revisión sistemática reciente también concluyen que el tratamiento ambulatorio tiene una baja tasa de necesidad de ingreso hospitalario $(<7 \%)$ y pocas complicaciones, sin olvidar el ahorro del coste sanitario ya apuntado por Lorente et al.

El valor real de la inclusión de antibióticos en el tratamiento de los pacientes con diverticulitis aguda leve solo se podrá establecer tras conocer los resultados de los ensayos clínicos prospectivos que se encuentran en curso. Aunque existen datos para pensar que en estos pacientes pudiera no ser necesario incluir antibióticos y que el tratamiento ambulatorio pudiese basarse en medidas dietéticas y analgésicas, por ahora, parece razonable mantener la antibioterapia en los protocolos de tratamiento.

Los datos de nuestro estudio indican que el tratamiento ambulatorio de la diverticulitis aguda leve en nuestro medio es seguro con el protocolo actual, ya que tuvo éxito en el $90,7 \%$ de los pacientes. El grado de cumplimiento de las pautas recomendadas fue satisfactorio. La incidencia de complicaciones durante el tratamiento fue baja, así como la necesidad de ingreso hospitalario. La eficacia del protocolo se basó en una adecuada selección de los pacientes.

Conflicto de intereses. Todos los autores declaran haber participado en la concepción y realización del trabajo que ha dado origen a esta publicación y que no tienen conflicto de intereses en su elaboración. 


\section{Referencias}

1. Elisei W, Brandimarte G, Tursi A. Management of diverticulosis: what's new? Minerva Med 2017; 108 (5): 448-463.

2. Aldoori WH, Giovannucci EL, Rockett HR, et al. A prospective study of dietary fibre types and symptomatic diverticular disease in men. J Nutr 1998; 128 (4): 714-719.

3. Sartelli M, Weber DG, Kluger Y, et al. 2020 update of the WSES guidelines for the management of acute colonic diverticulitis in the emergency setting. World J Emerg Surg 2020; 15 (1): 32.

4. Parks TG. Natural history of diverticular disease of the colon. Clin Gastroenterol 1975; 4: 53-69.

5. Shaikh S, Krukowski ZH. Outcome of a conservative policy for managing acute sigmoid diverticulitis. Br J Surg 2007; 94 (7): 876-879.

6. Ricciardi R, Baxter NN, Read TE, et al. Is the decline in the surgical treatment for diverticulitis associated with an increase in complicated diverticulitis? Dis Colon Rectum 2009; 52 (9): 1558-1563.

7. Murphy T, Hunt R, Fried M, Krabshuis JH. World Gastroenterology Organisation Practice Guideline: Diverticular Disease. Disponible en: https://www.worldgastroenterology.org/guidelines/global -guidelines/diverticular-disease. Consultado el 10 de enero de 2020.

8. Tursi A, Brandimarte G, Giorgetti GM, Elisei W. Mesalazine and/ or Lactobacillus casei in preventing recurrence of symptomatic uncomplicated diverticular disease of the colon: a prospective, randomized, open-label study. J Clin Gastroenterol 2006; 40 (4): 312-316.

9. De Korte N, Klarenbeek BR, Kuyvenhoven J, et al. Management of diverticulitis: results of a survey among gastroenterologists and surgeons. Colorectal Dis 2011; 13 (12): e411-e417.

10. Ambrosetti P, Grossholz M, Becker C, et al. Computed tomography in acute left colonic diverticulitis. Br J Surg 1997; 84 (4): 532-534.

11. Bone RC, Balk RA, Dellinger RP, et al. Definitions for sepsis and organ failure and guidelines for the use of innovative therapies in sepsis. The ACCP/SCCM Consensus Conference Committee. American College of Chest Physicians/Society of Critical Care Medicine. Chest 1992; 101 (6): 1644-1655.

12. Etzioni DA, Chiu VY, Cannom RR, et al. Outpatient treatment of acute diverticulitis: rates and predictors of failure. Dis Colon Rectum 2010; 53 (6): 861-865.
13. Biondo S, Fraccalvieri D. Enfermedad diverticular. En: Héctor Ortíz Aguado, editor. Cirugía colorrectal. Guía clínica de la Asociación Española de Cirujanos.2a ed. Madrid: Arán, 2012: 223-234.

14. Cirocchi R, Randolph JJ, Binda GA, et al. Is the outpatient management of acute diverticulitis safe and effective? A systematic review and meta-analysis. Techniques in Coloproctology 2019; 23 (2): 87-100.

15. Strate LL, Peery AF, Neumann I. American Gastroenterological Association Institute Technical Review on the Management of Acute Diverticulitis. Gastroenterology 2015; 149 (7): 1950-1976.

16. Stollman N, Smalley W, Hirano I, et al. American Gastroenterological Association Institute Guideline on the Management of Acute Diverticulitis. Gastroenterology 2015; 149 (7): 1944-1949.

17. Daniels L, Ünlü Ç, de Korte N, et al. Randomized clinical trial of observational versus antibiotic treatment for a first episode of CT-proven uncomplicated acute diverticulitis. Br J Surg 2017; 104: 52-61.

18. Mora López L, Ruiz-Edo N, Serra Pla S, et al. Multicentre, controlled, randomized clinical trial to compare the efficacy and safety of ambulatory treatment of mild acute diverticulitis without antibiotics with the standard treatment with antibiotics. Int J Colorectal Dis 2017; 32 (10): 1509-1516.

19. Emile SH, Elfeki H, Sakr A, Shalaby M. Management of acute uncomplicated diverticulits without antibiotics: a systematic review, meta-analysis, and meta-regression of predictor of treatment failure. Techniques in Coloproctology 2018; 22 (7): 499-509.

20. Azhar N, Kulstad H, Pålsson B, et al. Acute uncomplicated diverticulitis managed without antibiotics-difficult to introduce a new treatment protocol but few complications. Scandinavian J Gastroenterol 2019; 54 (1): 64-68.

21. Lorente L, Cots F, Alonso S, et al. Tratamiento ambulatorio de la diverticulitis aguda no complicada: impacto sobre los costes sanitarios. Cir Esp 2013; 91 (8): 504-509.

22. Van Dijk ST, Bos K, de Boer MGJ, et al. A systematic review and meta-analysis of outpatient treatment for acute diverticulitis. Int J Colorectal Dis 2018; 33 (5): 505-512. 